\title{
Clinical Complete Response
}

National Cancer Institute

\section{Source}

National Cancer Institute. Clinical Complete Response. NCI Thesaurus. Code C123574.

The disappearance of all signs of cancer based on physical exam, such as palpation. 